\title{
Cardiac PET/MRI enters the clinical arena! Finally...
}

\author{
C. Rischpler, MD, ${ }^{\mathrm{a}}$ N. Langwieser, $M D,{ }^{\mathrm{b}}$ and S. G. Nekolla, $\mathrm{PhD}^{\mathrm{a}}$ \\ a Nuklearmedizinische Klinik des Klinikums Rechts der Isar, Technische Universität München, \\ Munich, Germany \\ b Medizinische Klinik des Klinikums Rechts der Isar, Technische Universität München, Munich, \\ Germany
}

Received Sep 2, 2016; accepted Sep 2, 2016

doi:10.1007/s12350-016-0671-4

\section{See related article, pp. 785-794}

PET/MRI has been around for about 5 years now. Our institution was lucky enough to be the first place in the world where this "infernal machine" (also dubbed, "the beast" by the last author) was installed. Early after installation, first publications came out that described technical characteristics of this scanner. ${ }^{1}$ Soon after, the first paper comparing PET/MRI to PET/ $\mathrm{CT}$ in oncological patients ${ }^{2}$ and first manuscripts with the aim to define PET/MRI specific protocols were published. ${ }^{3}$ Also, a multiparametric approach trying to fully exploit the potential of this scanner was released within the first year after installation. ${ }^{4}$ Interestingly, even before the first original article dealing with hybrid PET/MRI in cardiovascular diseases was published, a review article by our group was released which pointed out the complexity of simultaneous cardiac PET/MRI, gave valuable ideas on the potential use of this scanner, and sought for the still illusive key application in cardiovascular diseases. ${ }^{5}$ Finally, in 2013 the first original article that solely described the feasibility of simultaneous cardiac ${ }^{18}$ F-FDG PET/MRI in patients after acute myocardial infarction was released. ${ }^{6}$ Following this article, there was a series of case reports ${ }^{7,8}$ and original articles ${ }^{9,10}$ - the latter mainly exploiting PET/ MRI as a research tool to have the ideal assessment of

Reprint requests: Christoph Rischpler, Nuclear Medicine, Technical University Munich, Munich, Germany; rischpler@gmail.com J Nucl Cardiol 2018;25:795-6.

$1071-3581 / \$ 34.00$

Copyright (c) 2016 American Society of Nuclear Cardiology. the left ventricular function (also for follow-up examinations with regards to outcome analysis) and the molecular signal by PET at the same time. Still, however, an original article evaluating cardiac hybrid PET/ MRI for a dedicated question that plays an important role in daily clinical routine was not released. In this issue of the Journal of Nuclear Cardiology, it is the first time (to the best of our knowledge) that PET/MRI has been evaluated for this purpose. Nensa et al. investigated the potential of hybrid ${ }^{18} \mathrm{~F}-\mathrm{FDG}$ PET/MRI in patients with the suspicion of myocarditis. Myocarditis is a disease which is characterized by an inflammation of cardiac tissue and which may be associated with a high morbidity and mortality. The cause of this inflammation is diverse, e.g., viral, bacterial, toxic, autoimmunogenic, or post transplantation. ${ }^{11}$ The current gold standard for the definitive diagnosis of myocarditis is endomyocardial biopsy, which, however, suffers from limited sensitivity due to sampling errors, a high complication rate (as high as about 6\%), and a disputable histological analysis (so-called Dallas criteria). ${ }^{12}$ It is because of these reasons that an improved diagnostic workup for patients with the suspicion of myocarditis is necessary. These days, it is daily clinical practice that those patients are treated based on morphological imaging using cardiac MRI. To standardize and to guarantee a certain quality of MR imaging and reporting, recommendations on the current state-of-the use of cardiac MRI for myocarditis have been developed, the so-called "Lake Louis Criteria". ${ }^{12}$ These criteria include at least edema (T2-weighted imaging), hyperemia (early gadolinium enhancement), and necrosis/scar (late gadolinium enhancement) imaging. Using these parameters, the sensitivity, specificity, and negative predictive values for the diagnosis of myocarditis with endomyocardial biopsy as the gold standard are 67\%, 91\%, and 69\%, 
respectively. ${ }^{12}$ Besides the relatively low sensitivity, the disadvantage of cardiac MRI for the diagnosis of myocarditis is that it is a rather morphological imaging modality with indirect assessment of information regarding disease activity from a physiological perspective. Hybrid ${ }^{18}$ F-FDG PET/MRI may help to overcome these shortcomings as increased glucose metabolism of cardiac tissue (based on the premise of a successful suppression of physiological myocardial uptake) represents active inflammation. But also in terms of imaging-guided endomyocardial biopsy it might improve sensitivity, whereas targeting areas of late gadolinium enhancement on cardiac MRI alone had not. ${ }^{13}$ PET/MRI may thus not only be useful to assist in the diagnosis of acute but also persisting viral myocarditis or virus-negative inflammatory cardiomyopathy. Furthermore it may allow to guide and monitor treatment response. Nensa et al. prospectively evaluated for the first time integrated ${ }^{18} \mathrm{~F}$-FDG PET/MRI in 65 patients with suspected myocarditis. When using a variant of the "Lake Louise Criteria" as reference, ${ }^{18}$ F-FDG PET had a sensitivity and specificity of $74 \%$ and $97 \%$ for the diagnosis of myocarditis. Also, ${ }^{18} \mathrm{~F}$ FDG PET demonstrated a high overall spatial agreement with cardiac MRI $(K=0.73)$. The authors concluded that ${ }^{18}$ F-FDG PET is in good agreement with cardiac MRI findings. The manuscript also revealed one major disadvantage of ${ }^{18}$ F-FDG PET: in about $12 \%$ of the patients, PET data could not be used for further analysis because patients demonstrated physiologic cardiomyocytic ${ }^{18} \mathrm{~F}$-FDG uptake possibly superimposing glucose metabolism caused by active inflammation. To overcome this issue, novel PET tracers, which specifically target inflammatory mechanisms, are necessary. The next step to evaluate this novel imaging approach would be to compare combined ${ }^{18}$ F-FDG PET/MRI data vs. MRI data alone with a group of raters, who have access to all clinical data (including endomyocardial biopsy, lab results, ECG, imaging data, etc.) as gold standard. Also, a prospective study using ${ }^{18} \mathrm{~F}$-FDG PET to guide and monitor treatment would be of high clinical interest. Still, the present paper is of clinical relevance as it is the first to evaluate hybrid ${ }^{18} \mathrm{~F}$-FDG PET/MRI in myocarditis and as it may pave the way for future cardiac imaging studies with PET/MRI (and novel tracers) as a powerful novel diagnostic tool.

\section{References}

1. Delso G, Furst S, Jakoby B, Ladebeck R, Ganter C, Nekolla SG, et al. Performance measurements of the Siemens mMR integrated whole-body PET/MR scanner. J Nucl Med. 2011;52(12):1914-22.

2. Drzezga A, Souvatzoglou M, Eiber M, Beer AJ, Furst S, MartinezMoller A, et al. First clinical experience with integrated wholebody PET/MR: Comparison to PET/CT in patients with oncologic diagnoses. J Nucl Med. 2012;53(6):845-55.

3. Martinez-Moller A, Eiber M, Nekolla SG, Souvatzoglou M, Drzezga A, Ziegler S, et al. Workflow and scan protocol considerations for integrated whole-body PET/MRI in oncology. J Nucl Med. 2012;53(9):1415-26.

4. Takei T, Souvatzoglou M, Beer AJ, Drzezga A, Ziegler S, Rummeny EJ, et al. A case of multimodality multiparametric $11 \mathrm{C}$ choline PET/MR for biopsy targeting in prior biopsy-negative primary prostate cancer. Clin Nucl Med. 2012;37(9):918-9.

5. Rischpler C, Nekolla SG, Dregely I, Schwaiger M. Hybrid PET/ MR imaging of the heart: Potential, initial experiences, and future prospects. J Nucl Med. 2013;54(3):402-15.

6. Nensa F, Poeppel TD, Beiderwellen K, Schelhorn J, Mahabadi AA, Erbel R, et al. Hybrid PET/MR imaging of the heart: Feasibility and initial results. Radiology. 2013;268(2):366-73.

7. Ibrahim T, Nekolla SG, Langwieser N, Rischpler C, Groha P, Laugwitz KL, et al. Simultaneous positron emission tomography/magnetic resonance imaging identifies sustained regional abnormalities in cardiac metabolism and function in stress-induced transient midventricular ballooning syndrome: A variant of Takotsubo cardiomyopathy. Circulation. 2012;126(21):e324-6.

8. Langwieser N, von Olshausen G, Rischpler C, Ibrahim T. Confirmation of diagnosis and graduation of inflammatory activity of Loeffler endocarditis by hybrid positron emission tomography/magnetic resonance imaging. Eur Heart J. 2014;35(36):2496.

9. Rischpler C, Langwieser N, Souvatzoglou M, Batrice A, van Marwick S, Snajberk J, et al. PET/MRI early after myocardial infarction: Evaluation of viability with late gadolinium enhancement transmurality vs. 18F-FDG uptake. Eur Heart J Cardiovasc Imaging. 2015;16(6):661-9.

10. Rischpler C, Dirschinger RJ, Nekolla SG, Kossmann H, Nicolosi $\mathrm{S}$, Hanus F, et al. Prospective evaluation of 18F-fluorodeoxyglucose uptake in postischemic myocardium by simultaneous positron emission tomography/magnetic resonance imaging as a prognostic marker of functional outcome. Circ Cardiovasc Imaging. 2016;9(4):e004316.

11. Liu PP, Mason JW. Advances in the understanding of myocarditis. Circulation. 2001;104(9):1076-82.

12. Friedrich MG, Sechtem U, Schulz-Menger J, Holmvang G, Alakija $\mathrm{P}$, Cooper LT, et al. Cardiovascular magnetic resonance in myocarditis: A JACC white paper. J Am Coll Cardiol. 2009;53(17):1475-87.

13. Yilmaz A, Kindermann I, Kindermann M, Mahfoud F, Ukena C, Athanasiadis A, et al. Comparative evaluation of left and right ventricular endomyocardial biopsy: Differences in complication rate and diagnostic performance. Circulation. 2010;122(9):900-9. 